
profesional

\title{
Intervenciones del Trabajo Social desde el poder judicial en situaciones de violencia familiar.
}

\section{Reflexiones y proyecciones a partir de una situación singular}

Social Work interventions from the judiciary in contexts of family violence

Viviana Elisa Pascual

Licenciada en Trabajo Social

(Universidad Nacional de Rosario)

Magister en Trabajo Social

(Universidad Nacional de Entre Ríos)

Correo: vivianapascual@yahoo.com

Silvana Martino

Licenciada en Trabajo Social

(Universidad Nacional de Rosario)

Magister en Ciencias Sociales (FLACSO)

Doctora en Humanidades y Artes, mención en Antropología (Universidad Nacional de Rosario)

Correo: silvanamartino2012@gmail.com 


\section{Resumen}

El presente artículo tiene como propósito recuperar una de las situaciones que requieren la intervención del trabajador social a diario, y cómo de esa cotidianeidad se llega a una naturalización/rutinización de las nociones, posiciones y fundamentos que atraviesan la práctica profesional de manera decisiva. Se propone desandar las acciones y omisiones que se fueron realizando a lo largo de una situación de violencia familiar que llega al poder judicial y requiere imperiosamente ser abordada. Desde allí, intentamos re preguntarnos acerca de nuestro quehacer y el de les agentes implicades en dicho proceso, con la certeza que re pensarnos e interpelarnos no sólo nos permite producir conocimientos acerca de nuestras prácticas y experiencias, sino reafirmar nuestra posición ética política en el acceso a la justicia de los vulnerados, en este caso, mujeres y niñes víctimas de violencia familiar.

Palabras clave

Intervención, Visita domiciliaria, Violencia.

\section{Abstract}

The purpose of this article is to recover one of the situations that require the intervention of the social worker on a daily basis, and from that daily life, a naturalization / routinization of the notions, positions and foundations that decisively cross the professional practice is reached. That is why we propose to retrace the actions and omissions that were carried out throughout a situation of family violence that reaches the judiciary and urgently needs to be addressed. From there, we try to re-ask ourselves about our work, and that of all the agents involved in said process, with the certainty that by rethinking and questioning ourselves, it not only allows us to produce knowledge about our practices and experiences but also to reaffirm our ethical political position in access to justice for the violated, in this case, women and children victims of family violence.

\section{Keywords}

Intervention, Home visit, Violence. 


\section{Introducción}

Algunas de las situaciones por las que se nos solicita con mayor frecuencia intervención a les trabajadores sociales insertos en el Equipo Único de Trabajo Social (en adelante, EUTS) en el ámbito de familia en el poder Judicial están referidas a violencia familiar. Estas llegan a la instancia judicial a través de la oficina de Violencia de género y Violencia Familiar instalada en el mismo ámbito del tribunal. Desde allí, se despliega un sinfín de trámites/acciones que incluyen: escritos que deben circular por diversas oficinas, relatos que les denunciantes deben repetir reiteradas veces y esperas por parte de les mismes sujetes que denuncian que conllevan, en esta primera instancia, más de una mañana. Estos “trámites”, como es sabido, involucran sujetes, relatos, decisiones y resoluciones.

También, es sabido que, debido a que se trata de situaciones complejas, se torna imposible rigidizar los modos de intervención de les profesionales -al menos abocados a esta temática en el ámbito judicial- en modelos estándar. Cada situación implica singularidades, tales como historias personales y colectivas, referencias institucionales y familiares, temores, soledades, etc., las cuales hay que considerar y visibilizar a la hora de intervenir en tal complejo y delicado escenario.

Tal como lo plantea González Saibene (2020):

(...) trabajar con la idea de complejidad nos permite pensar en múltiples y diversos escenarios. Estos cambian de acuerdo a cómo se mueven distintos actores. Esto también forma parte de lo que entendemos por estrategia. Una estrategia tiene que tener la capacidad de poder leer distintos actores y distintos escenarios que se mueven permanentemente, por lo tanto un programa no puede ser nunca normativamente establecido, sino estratégicamente pensado. Es decir, tiene que tener la capacidad de moverse, movilizarse en la medida en que los actores y escenarios cambian (2020:3).

Sumergidas en la lógica de desentrañar (nos) nuestras propias intervenciones en primer lugar, y luego desde nuestra mirada como operadores del ámbito judicial, nos detendremos en una situación de violencia singular abordada a partir del pedido de intervención de un 
Juez de turno a Trabajo Social, con el objeto de repensar interrogantes, nuevas estrategias, y construir posibles alternativas al abordaje de situaciones ligadas a la violencia de género y violencia familiar.

Conscientes de la ruta crítica que transitan las mujeres en situación de violencia, debemos detectar los obstáculos, pensar en estrategias para modificar prácticas y remover aquellos que se presentan en el ámbito de la justicia y dificultan hacer efectivos sus derechos. Partimos de la necesidad de modificar prácticas atravesadas por lo cultural que influyen al momento de abordar, asesorar, requerir o decidir un caso vinculado a la temática de género, a la par de dar a conocer los derechos humanos de las mujeres contenidos en nuestro bloque normativo y demás instrumentos, para que se vean reflejadas en nuestras prácticas cotidianas (Leiro, 2019:16).

Cabe mencionar que en lo que respecta a las carátulas de los expedientes que son derivados de los Tribunales Colegiados de Familia, llevan todos la denominación de Violencia Familiar, sin discriminar si se refiere a violencia de género, maltrato infantil, violencia doméstica, etc.

De este modo, y a partir de la descripción detallada de esta situación, se pondrán en tensión las nociones en juego en torno a la solicitud de una técnica de actuación profesional del Trabajo Social -diría Tonon (2005)- como es la visita domiciliaria, atravesada con el adjetivo de urgente y/o inmediata. Para esto, nos iremos deteniendo en el relato de la denuncia para hilvanarlo con nociones teóricas que nos ayuden a reflexionar y construir posibles alternativas para dar respuestas que no repitan la vulneración del derecho denunciado.

Antes de ello, resulta necesario explicitar la posición epistemológica y política que sustenta nuestra práctica profesional, más allá de la noción de violencia en sí trabajada conceptualmente por una vasta y rica bibliografía.

Las situaciones que "llegan" a los tribunales bajo la denominación de denuncia de violencia tienen como foco situaciones de vulneración de derechos ligadas al ejercicio de la violencia física primordialmente, en la cual, de manera solapada y en algunos de sus argumentos, apare- 
cen mecanismos de violencia psicológica o moral, sexual y económica. No obstante, estas situaciones narradas en los textos de denuncias no pueden reducirse al hecho en sí. Es necesario comprenderlas desde miradas complejas, integrales, donde sea posible hacer intervenir otras nociones y otras vulneraciones. De este modo, no se reduce la situación a un solo aspecto ni sus posibilidades de respuesta:

Negarse a reducir la complejidad es una posición ética con implicancias políticas. Esto permite restaurar las singularidades, esto permite volver a retomar todo lo que tiene que ver con lo local, con lo diverso. La necesidad de restaurar las singularidades, lo local, lo diverso, es lo que nos va a permitir elaborar las estrategias o las resistencias según cómo sea el caso (González Saibene, 2020:3).

Por otro lado, como marco normativo, a partir del año 1997 se sanciona en la provincia de Santa Fe (Argentina) la ley 11.529 de Protección contra la Violencia Familiar. Conforme esta ley, el ámbito de protección de la misma abarca:

(...) a todas aquellas personas que sufriesen lesiones o malos tratos físicos o psíquicos por parte de alguno de los integrantes del grupo familiar. A los efectos de esta ley, entendiéndose por tal surgido del matrimonio o uniones de hecho, sean convivientes o no, comprendiendo ascendientes, descendientes y colaterales (Ley 11529, art.1).

En el año 2001 se reglamenta la mencionada ley mediante el Decreto 1.745/2001 que amplía el ámbito de aplicación de la misma definiendo a la violencia familiar como “(...) toda acción u omisión ejercida por un integrante del grupo familiar contra otro que produce un daño no accidental en lo físico, psíquico, sexual o patrimonial” (Blotta et al., 2011:311-312).

\section{La singularidad del caso}

Nuestra participación en la situación enunciada es convocada en el turno llamado de "urgencia". Allí, el propio secretario es quien manifiesta telefónicamente el interés y necesidad del Juez de que nos cons- 
tituyamos en el domicilio de la persona víctima de violencia familiar, de la cual y en nombre de ésta, una Señora había realizado la presente denuncia.

Allí mismo, se le solicita al secretario envíe el texto de la denuncia para conocer detalles básicos, en principio, como: nombres de las personas involucradas, relaciones entre ellas, domicilios denunciados, y luego, el relato sobre la situación de aquella persona que hizo la denuncia. Junto con esto, se solicita el decreto donde se pide nuestra intervención y allí encontramos:

Atento a los hechos expuestos previo requiérase al Equipo Único de Trabajo Social, que con carácter de urgente, se proceda a realizar un pormenorizado informe ambiental en el domicilio ... de Rosario, recabando información de los vecinos y todo dato de interés. Así mismo, dese intervención a la Dirección provincial de Niños respecto a "R" de 11 años y " $S$ " de 9 años que se domicilian en ... (el mismo domicilio mencionado más arriba) (texto de la denuncia citada).

En el texto de la denuncia realizada en la Oficina de Atención a las Víctimas de Violencia Doméstica y de Género del Ministerio Público a las $12.13 \mathrm{~h}$. , aparece como denunciante, el nombre de una señora, quien indica que es la delegada del Ministerio Nacional de Mujeres, Género y Diversidad de la Provincia de Santa Fe. Junto con esto se explicita su domicilio y su número de teléfono celular.

Esta señora, a quien llamaremos Rosa, dice haber realizado la misma denuncia en el Centro Territorial de Denuncias perteneciente a su propio domicilio hace tres meses atrás.

Rosa vuelve a exponer el relato de su denuncia. Esta se trata de dos hermanas, de 23 y 21 años, víctimas de violencia y abuso sexual por parte de su padre, con quien conviven. A su vez, también residen allí los hijos de éstas, nietos del agresor y su madre, una de las parejas de este señor.

Agrega que Ramón, como llamaremos al agresor, mantuvo desde hace muchos años, relación con dos hermanas, una de ellas madre de las mujeres mencionadas al comienzo. Una de estas dos hermanas con quien tuvo siete hijos, algunos mayores de edad, pudo "terminar" la 
relación con Ramón, al irse del barrio. No obstante, la otra quedó conviviendo con Ramón, sus hijes mayores y otros dos hijes menores, de 9 y 11 años, de quien además, se presume son víctimas de abuso sexual por parte de su padre. Expresa en el mismo escrito, además, que en sede penal se están realizando las investigaciones pertinentes y da el nombre de le fiscal que se ocupa de tal trabajo. En la sede arriba mencionada cuentan con testimonios de la ex pareja de Ramón, de dos de sus hijes, coincidentes con lo expuesto por Rosa en la presente denuncia y a su vez, cuentan con el testimonio de una trabajadora social perteneciente a una institución de referencia barrial, quien al intentar acercarse al domicilio fue amenazada con un arma de fuego, la cual porta Ramón. Sobre esto último, expone que utiliza el arma de fuego, un revolver, para amenazar a cualquier persona que haya querido ayudar a las víctimas.

Por todo lo expuesto, reza la denuncia, se solicita exclusión de hogar del Sr. Ramón y su consecuente prohibición de acercamiento. Que les niñes sean escuchados por S.S y por le Defensor General. Se dé intervención a la Dirección de Niñez. Solicita, además, ya hacia el final del texto, que la notificación de la medida de protección se realice mediante la Agencia de Investigación para la trata de personas y el Centro de Asistencia a la Víctima de Delitos Sexuales. Cabe resaltar aquí, que el pedido que se le realiza le Juez es la exclusión del hogar del agresor, y sobre este particular debía resolver le Magistrade. Finaliza el acta a las 12.50, llevando la firma de le Fiscal de dicha Oficina de Atención.

Frente a esta primera lectura, surgen algunos interrogantes tales como, ¿quién es esta señora que realiza la denuncia? ¿Qué relación tiene el organismo del que es delegada, con la situación planteada? ¿De qué se trata este organismo? ¿Trabajan en conjunto con otros organismos del estado? ¿Quiénes más conocen la situación y han intervenido? Respecto a esto, aparece el nombre y el apellido de la Trabajadora Social como así también el de la Institución en la que trabaja. Esto nos permite suponer que ya al menos hay tres instituciones interviniendo: Ministerio Nacional de las Mujeres, Género y Diversidad de la provincia de Santa Fe, el Poder Judicial- sede penal- y la institución barrial. 
Además, les niñes mencionados, ¿concurren a la escuela? Desde allí, ¿qué se conoce sobre la situación planteada? ¿Han intervenido?

¿En qué momento de la intervención podrían estar estas instituciones? ¿Lo harán de manera conjunta o no existe conexión entre ellas?

Y la madre de estes hermanes, víctimas de abuso y violencia, ¿̇reside allí? ¿Qué papel ocupa en la trama de dicho relato?

Otres familiares: ¿Quiénes acceden a esta vivienda? ¿Qué se conoce de elles?

Así se abrían múltiples interrogantes los cuales, a su vez, abrían a múltiples intervenciones posibles.

En ese momento, entendimos que lo primordial era, dada la gravedad de la situación planteada, dialogar con Rosa, primera actriz del relato.

En el transcurso de esa tarde, en la que recibimos el llamado telefónico del secretario de violencia para solicitar nuestra intervención, nos comunicamos también de manera telefónica con Rosa. Ella, quien se encontraba saliendo de los tribunales, expresa que se siente abrumada y sorprendida por la decisión de le Juez. Aquello que abrumaba a Rosa era no haber obtenido la exclusión de hogar a modo de medida autosatisfactiva. Al preguntarle cuál era la razón para esto, refiere que hace muchos años conocen e intervienen en la situación, y que llegado este punto habían pensado y diseñado una estrategia de manera conjunta con el Área de Género de la Municipalidad de Rosario y con el Director del Centro de Acceso a la Justicia, de quienes da sus nombres, para abordar de manera conjunta y rotunda la situación: es decir, se trataba de notificar al victimario de la exclusión de hogar, y simultáneamente, a través del Centro de Asistencia a las Víctimas, atender a las hermanas, para las cuales además, se había previsto un lugar de alojamiento en un centro de protección. Junto con esto, consulta sobre la posibilidad de pasar nuestro número de celular personal, desde donde nos estábamos comunicando para que alguno de estos profesionales intervinientes se ponga en contacto con nosotras.

Insiste y remarca que de llevarse adelante la intervención ordenada por le Juez (visita domiciliaria) al Equipo Único, solo se lograría poner 
en sobre aviso al agresor, y consecuentemente, el fracaso de la estrategia sostenida inter institucionalmente.

Aquí aparece un nudo interesante para detenernos a considerar, dado que éste a su vez, reviste algunas contradicciones que tensan la situación y sus posibilidades de intervención: se trata del pedido de intervención de le Juez, enmarcado o encerrado en la visita domiciliaria, por un lado; lo manifestado por quien realiza la denuncia sobre los peligros de realizarse esta medida, por otro; y finalmente, la ética profesional puesta en juego de aquel a quien se convoca a realizarla.

\section{Fundamentos teóricos conceptuales}

La propia historia del trabajo social, tal como lo afirma Valentín González Calvo “(...) está inevitablemente vinculada a la Visita Domiciliaria. Ésta que aún encierra mucho de nuestra especificidad, ha ido transformando sus objetivos y sus modos" (2003:2). Desde aquellas visitadoras amigables de comienzo del siglo XX como las definía Mary Richmond (Tonon, 2005), como inicio e intento de profesionalización de nuestro quehacer hasta nuestros días, es posible encontrar otras razones y fundamentos para la llegada de profesionales a los lugares donde habita el sujeto de nuestras intervenciones.

Este acercamiento, el estar allí, abre un sinfín de posibilidades de intervención a partir de otra escucha, de la construcción de otros relatos, de la observación de los contextos en dónde éstos se producen. Como es sabido, esto conlleva en muchas ocasiones, que las situaciones que "aparecen" como simples o reducidas a una sola cuestión, se complejicen y se abran a nuevas demandas y relatos. Lejos de suponer que lo que uno ve, escucha y siente es la "pura" y "verdadera" realidad, la cual se muestra automáticamente tal cómo es con solo llegar hasta dónde suceden las cosas, sostenemos que esa realidad, ese relato se construye, se negocia, se acuerda, se oscurece y/o puede mostrarse sólo una parte. Valentín Gonzalez Calvo, describe distintos escenarios en los que se realiza la visita domiciliaria con sus consecuentes límites o potenciadores de esta intervención. En un contexto asistencial, de acuerdo al recurso puesto en juego, el trabajador social en las visitas domiciliarias puede comprobar, confirmar, conocer un: 
(...) buen número de datos necesarios para comprender a fondo la situación y realizar los informes oportunos que se pudieran derivar de la solicitud del usuario. De común la familia ante la expectativa de recibir algún tipo de ayuda favorece que el trabajador social se acerque a su domicilio (Gonzalez Calvo, 2003:6).

No obstante, este mismo autor menciona la existencia de la otra cara de la moneda del contexto asistencial, la del control, ligada a la visita en el domicilio. Si se da una prestación material, en especie o en servicio hay que "controlar" que ésta sea destinada al fin previsto. Aquí, remarca, “(...) cuando surgen dos problemas, de una parte, la definición del papel profesional; y de otra, la dificultad de la familia para posicionarse ante una u otra dimensión del profesional (ayuda o control)" (Gonzalez Calvo, 2003:6).

Estas circunstancias influyen sobremanera en el transcurso de la/s visita/s toda vez que la familia no tiene claro si el profesional va a inspeccionarles, ayudarles, vigilarles.

Esto puede suceder cuando no media una demanda directa de la familia sino que la visita en el domicilio está relacionada a la petición que otres hacen (juez, fiscal, etc.) de "constatación, supervisión, recogida de información, seguimiento, etc. acerca de dicha familia en cuestión. Cuando la visita se hace bajo este encargo, la interacción profesional-cliente resulta bastante embarazosa" (Gonzalez Calvo, 2003:6) y hasta se podría decir, éticamente cuestionable. "Por su parte la familia desconfía del profesional y a la vez podría llegar hasta personalizar a éste como el artífice de las medidas negativas que obren sobre ella" (2003:6). Sigue el mismo autor,

(...) los que nos hemos encontrado en este tipo de vicisitudes, nos habremos dado cuenta que nosotros, sin quererlo, somos la cara de instituciones anónimas que a vista de la familia le están perjudicando, y es sobre el profesional a quien dirigen todas sus iras. Realmente en este contexto se pueden dar situaciones extremas de agresión y violencia contra el trabajador social, por lo que es conveniente tomar las medidas de seguridad que se estimen oportunas tendientes 
a prevenir daños contra la persona del profesional o de sus bienes (Gonzalez Calvo, 2003:6).

Nos interesa detenernos y poner especial énfasis en esta situación posible y extrema descripta más arriba, dado que está relacionada a las visitas domiciliarias como intervención de les profesionales del Trabajo Social, representando al Poder Judicial, en el marco de una denuncia de violencia familiar.

Si bien el pedido de intervención de "realícese un amplio y detallado informe ambiental en el domicilio denunciado" "o en el de las partes”, surge a partir de una demanda concreta, en general, por la víctima, también puede suceder, como el caso que aquí nos convoca, que otros sean los portavoces de tal denuncia, como ser una institución, un profesional, algún vecine o algún familiar. La llegada al domicilio de la víctima (la cual anoticiada de nuestra intervención puede estar esperándonos) o del victimario, quien desconoce que sobre él pesa una "denuncia” (con toda la carga simbólica que ésta representa) o al domicilio donde ambos aún residen, víctima y victimario, se constituyen en distintos escenarios de posibles intervenciones.

Y es aquí, ponderando estos escenarios, donde la intervención profesional debe basarse en la lectura de la situación, en la evaluación de la gravedad de ésta y de los riesgos a los que estaría exponiendo a las víctimas con la sola presencia física (institucional, ya que encarnamos simbólicamente a la institución a la que en ese momento representamos) en el domicilio. De darse estos riesgos o posibles situaciones de vulnerabilidad sobre todo para la/s víctima/s, se complejiza y agudiza el trabajo. Ya que este no se limita o culmina con la visita en el domicilio (donde muchas veces ni siquiera se encuentran las personas a las que debemos entrevistar, al momento de nuestra visita), sino que se debe comenzar a construir cartografías vinculares o institucionales que puedan brindar no solo los datos acerca de la situación denunciada sino que puedan a su vez, constituirse en soporte de la situación y del cambio de ésta a partir de una decisión judicial (excluir con la fuerza pública al agresor; prohibición de acercamiento a las víctimas, etc.).

En este punto y frente a estas situaciones, lo territorial excede a lo ambiental (entendiendo a este solo como el domicilio). En el territo- 
rio, llamado en el ámbito de la gestión de política como lo próximo, lo local, es posible construir y pensar estrategias que incluyan a otros (instituciones, familiares, etc.) para la constitución de acuerdos inter sectoriales (municipalidad, provincia y nación) con distintos organismos, de manera horizontal (centro de salud- municipal o provincial; programa de atención a la violencia de género- teléfono verde, dependiente de Desarrollo Social de la municipalidad, Dirección General de Infancias de la Municipalidad; Desarrollo Social dependiente del gobierno de la Provincia; Vecinal, Centro comunitario), etc., para así formar redes de atención, información y acceso.

Entonces, volviendo a la situación planteada,

¿Se podría haber sostenido entrevista en sede judicial con la denunciante a fin de tener mayores detalles y precisiones a la denuncia recientemente realizada, antes de evaluar las medidas a solicitar?

Pero, ¿por qué esta persona se acerca sola a realizar tal denuncia, desprovista de documentación que dé cuenta de la institución a la que representa e informes de lo intervenido hasta allí?

Una vez obtenida mayor información, la cual no consta ni se entrevé en la denuncia, ¿se podría comunicar con los organismos nombrados por la denunciante a fin de acordar estrategias en conjunto o al menos no entorpecer la diseñada por estos otros?

Tener en consideración que si el agresor y la víctima conviven en el mismo domicilio, el acercarnos a éste en nombre del Poder Judicial, podría poner en mayores riesgo a las víctimas, durante o posterior a la entrevista realizada allí, y al mismo profesional presente en dicha situación.

Reconsiderar lo que se llama urgencia y qué es lo que permite considerarla bajo cada una de las consecuencias y ventajas posibles en torno a ésta.

¿Qué se espera pueda aportar "la visita domiciliaria”, en semejante contexto de riesgo que fue descripto por la denunciante?

No obstante estos interrogantes, las intervenciones desde el Equipo Único continuaron en la misma línea que la "ordenada". Se solicitó a la autoridad policial del Tribunal a fin de poder ser trasladadas a terri- 
torio con movilidad oficial (teniendo en cuenta lo grave y riesgosa de la situación denunciada). En primer lugar, se nos argumentó no disponer del móvil policial, pero con el transcurso de los minutos y luego de intensas comunicaciones telefónicas, el recurso estuvo disponible: se dispuso personal femenino y auto para realizar la medida. A su vez, de manera paralela, nos comunicamos, luego de hablar con Rosa, con la Jueza de turno (quien había solicitado nuestra intervención). Esta expresa su posición frente a la situación denunciada, haciendo hincapié en las irregularidades y falencias de la propia presentación. Advertida de los riesgos que conlleva nuestra intervención en el domicilio, plantea que no nos constituyamos allí para nuestro resguardo.

Sin embargo, ya el móvil policial estaba en marcha hacia el domicilio. Una vez en la esquina de éste decidimos acercarnos a la institución barrial (Centro de Salud) ubicada en la misma esquina de la vivienda donde reside el grupo familiar en cuestión, donde se desempeña la Trabajadora Social citada en la denuncia. Al llegar allí, solo se encontraba el personal administrativo, dado que se trataba de las últimas horas de la tarde. Éste, de manera muy cautelosa, no brinda demasiados datos al respecto y nos solicita nos contactemos con les profesionales, les cuales asistirían al otro día por la mañana. Así mismo, nos comunicamos telefónicamente al celular personal de la trabajadora social, quien nos advierte sobre la peligrosidad de la situación. Solicita no hablar del tema allí y no ser nombrada, ya que el agresor se encuentra armado y ella misma fue amenazada. Se acuerda una reunión para el día posterior, contando para entonces, con les demás profesionales que habían intervenido (pediatra, clínica/o, psicóloga/o) y la documentación necesaria (historia clínica, denuncias, informes interdisciplinarios, etc.).

Al día siguiente, por la mañana, el Coordinador del Centro de Acceso a la Justicia, se comunica a nuestro celular personal, preguntándonos si se había realizado la medida solicitada. Agrega no solo su disconformidad sobre ésta y agradece que ésta no se haya llevado adelante, para así poder proseguir con la intervención diseñada. Ésta se trataba de llegar al domicilio todas las instituciones intervinientes y "sacar" a les hijes del agresor para ser alojadas en un Centro de Protección Integral para mujeres víctimas de Violencia. 
Tres días después de haberse recibido la denuncia, el expediente con la presentación realizada por Rosa que había ingresado en un turno de urgencia, luego de un sorteo realizado por la Mesa de Entrada, llega al Juzgado que deberá continuar el trámite.

Insistimos, tres días después de "la demanda de intervención urgente", de manera disruptiva, el Juez vuelve a "ordenar" que se realice la medida solicitada en el Juzgado de turno en su momento. Luego de intentar infructuosamente dialogar con éste, se comunica el Coordinador del Centro de Acceso a la Justicia, para dar cuenta de lo acordado por todas las instituciones intervinientes, en relación a la situación denunciada. A pesar de lo informado por el Coordinador, se solicita con urgencia, el informe de la visita en el domicilio, de la trabajadora social.

Acá nuevamente aparece el adjetivo que suele acompañar a cada uno de los pedidos de intervención al Trabajo Social, la urgencia, en muchos casos de manera acrítica. Noción que no permite, en el contexto en el que aparece, preguntarse, detenerse, interpelar. Podríamos sostener que las nociones de urgencia e inmediatez (Minnicelli, 2020) se confunden, lo cual limita la intervención y sus posibilidades-.

Esta inmediatez puede devenir en apuro, provocando acciones sin la necesaria información, sin los recursos y sin pensarlas con detenimiento para que éstas no resulten insuficientes, fragmentadas y muchas veces riesgosas para quienes realizan una denuncia.

Por esto, pensar la intervención, no desde la inmediatez, sino desde la complejidad (la cual seguramente contempla la urgencia), puede conducirnos a la reconstrucción de los recorridos y mapas institucionales que les propies actores sociales construyeron antes de arribar a una instancia judicial e incluso convocar a partir de éste, a un trabajo transversal, interdisciplinario e interinstitucional, tendiente a provocar otro resultado o desenlace. Esto puede constituirse en nuestro aporte distintivo plasmado en "un informe socioambiental".

\section{Reflexiones finales, a modo de conclusiones abiertas}

Volviendo al caso presentado, lo que las instituciones intervinientes, desde hacía tiempo, en dicha situación (las cuales, a través de sus 
profesionales, y agentes, habían analizado la situación y pensado la mejor estrategia de intervención), venían a solicitar a la Justicia, era la exclusión de hogar del agresor. Ésta era necesaria, para que en caso que el agresor saliera en libertad (ya que había sido detenido), tuviera una limitación legal, para regresar al domicilio.

Con todo lo relatado por quien coordinara el operativo, el cual había sido llevado adelante con éxito, nos preguntamos: ¿para qué era necesario el informe ambiental? Ya lo habíamos preguntado al momento de la denuncia, ya que con sobrantes indicadores de riesgo y de violencia, no quedaban claros que otros elementos o información se necesitaba para ordenar la exclusión. Y nos volvimos a hacer la pregunta, tres días después, luego que las instituciones intervinientes habían llevado adelante la estrategia diseñada.

Coincidiendo con Tonón, "el lugar instituido de las visitas domiciliarias en las organizaciones, se relaciona más con el cumplimiento de procedimientos administrativos que con la generación de alternativas de solución de los problemas sociales" (2005:69), lo cual nos interpela en la necesidad de "desburocratizar esta situación".

Como contracara a estas solicitudes descontextualizadas, revalorizamos y ponemos fuertemente el énfasis en la noción de territorio, no como el espacio de realización de la "visita domiciliaria", sino como el "lugar de identificación e identidad, el lugar de la producción de vínculos, el lugar donde el relato de las víctimas de violencia”, encuentra anclajes que la resguarden.

En este sentido, citando el estudio sobre La violencia de género y el acceso a la justicia de las mujeres en la provincia de Santa Fe - Argentina, realizado por un grupo de profesionales del Centro de Estudios sociales y culturales para la comunidad en el marco del Observatorio de Violencia de Género, adherimos a la necesidad de rescatar las voces de las mujeres afectadas por esta problemática, ya que ellas mismas señalan que:

(...) los tribunales deberían estar en los barrios evitando los continuos traslados con las dificultades de todo tipo que esta acción implica. Este último punto permite rescatar el rol que deberían asumir las Defensorías Barriales en la agilización de trámites, de modo 
de profundizar los procesos de descentralización que implicaría que solo llegara a Tribunales casos muy específicos (Blotta, Fullone, Fuentes, Galizia, Gancedo, 2011:322-323).

Junto con esto, y a la idea de red multinivel, o la conformación de redes, la descentralización territorial del propio tribunal cobra hoy una relevancia inusitada y provocadora de nuevas intervenciones.

Las Defensorías zonales son actualmente esos espacios territorializados, cercanos tanto a los ciudadanos que demandan información, asesoramiento y acompañamiento como de las instituciones estatales locales.

Las defensorías zonales se erigen ya como pruebas cabales de una descentralización exitosa al lograr acercar la Justicia al más vulnerable ya no en la sede tribunalicia sino allí donde el más vulnerable tiene su centro de vida (...) volviendo más accesible el servicio a personas que de otro modo no tendrían posibilidad de tenerlo, patrocinando judicialmente ciertos asuntos de jurisdicción voluntaria (De luca, 2003:103).

Las defensorías zonales, con sus funcionarios cercanos a la realidad de los sectores vulnerables dejan de lado la perspectiva adversarial y promueven soluciones integrales, trabajando interdisciplinariamente a partir de mecanismos de mediación y conciliación (Casiello, Fornasari, 2016:428).

Entonces, ¿por qué no pensar que el eje de esta nueva mirada o perspectiva pueda estar centrada en las mismas estructuras que el propio poder judicial ideó hace 30 años? porque no potenciar estos espacios para poder diseñar estrategias interdisciplinarias e interinsitucionales para abordajes cada vez más complejos de las situaciones sociales en las que la violencia familiar, la violencia de género no es su excepción.

\section{Referencias bibliográficas}

Bastianelli, Liliana (2004). Una mirada al interior de las defensorías zonales. Revista del Colegio de Magistrados y funcionarios del poder judicial de la provincia de Santa Fe, (4), 396- 403. 
Blotta, María Florencia; Fullone, Beatriz; Fuentes, Adriana; Galizia, Beatriz, y Gancedo, Nora (2011). La violencia de género y el acceso a la justicia de las mujeres en la provincia de Santa Fe- Argentina. Revista de Estudios Jurídicos, 15(22), 309-324.

Casiello, María Victoria; Fornasari, Sergio (2016). Las defensorías Zonales: verdaderos agentes del cambio social en Revista del Colegio de Magistrados y funcionarios del poder judicial de la provincia de Santa Fe, (6), 422-429.

De Luca, Marcela (2003). Una realidad desafiante. Revista del Colegio de Magistrados y funcionarios del poder judicial de la provincia de Santa Fe, (3), 98-105.

González Calvo, Valentín (2003). La visita domiciliaria, una oportunidad para el conocimiento de la dinámica relacional de la familia. Revista Servicios Sociales y Política Social, (61), 63-86.

González Saibene, Alicia (2020). Los desafíos del trabajo social hoy. $1^{\circ}$ Congreso Virtual de Ciencias Sociales. Rosario, Argentina. Facultad de Ciencia Política y Relaciones Internacionales, Universidad Nacional de Rosario, Mimeo

Leiro, María Pía (2019). Perspectiva de Género en la administración de justicia. Los derechos de las mujeres. Cuaderno $N^{\circ} 14$ - FUNDEJUS. Buenos Aires, Fundejus.

Ley $\mathrm{N}^{\circ} 11529$-Violencia familiar (2001). Santa Fe, Argentina.

Minnicelli, Mercedes (2020). Programa de Formación "Voces y Comunidades". Mar del Plata, Argentina, Facultad Psicologia, Universidad Nacional de Mar del Plata.

Pascual, Viviana; Martino, Silvana (2020). Intervenciones Irreverentes en el ámbito judicial. Propuestas de cambio al interior y desde el Trabajo Social. Revista Margen, (97).

Sousa Minayo, M.aría Cecilia (2013). La artesanía de la investigación cualitativa. Buenos Aires, Argentina, Lugar editorial.

Tonon, Graciela (comp.) (2005). Las técnicas de actuación profesional del Trabajo Social. Buenos Aires, Argentina, Espacio editorial.

Recibido: 20/07/2021

Aceptado: 27/09/2021 